\title{
Factors associated with suicide among African American adult men: a systematic review of the literature
}

\section{Keywords}

\section{Suicide}

Factors

African American/ Black

Men/males
K.L. Rowell, PhD, MPH Moody, AL, USA

B.L. Green, PhD

H. Lee Moffitt Cancer Center, Tampa, FL, USA

\section{J. Guidry, PhD}

Texas A \& M University,

TX, USA

\section{J. Eddy, D.Ed}

The University of North

Carolina, Greensboro, NC, USA

E-mail: kyrelrowell@yahoo.com

Online 21 November 2008

\section{K.L. Rowell, B.L. Green, J. Guidry and J. Eddy}

Abstract

The suicide prevalence rate among African American men continues to be lower than Caucasian men, yet an increasing rate of suicide completions among African American men is evident. If the current upward trend persists, the suicide rates among African American men will rival that of Caucasian men. Central to suicide prevention in African American men is an increased examination and understanding of risk and protective factors. Therefore, the purpose of this study was to systematically and critically review the existing literature regarding factors associated with completed suicide among African American adult men.

Using the Garrard matrix method, 266 potential articles were identified through the search of 14 electronic databases and numerous reference lists. Quality assessment criteria were devised to evaluate the studies' merit and credibility. After applying inclusion and exclusion criteria, nine empirical studies specifying suicide risk and protective factors were identified. Within these nine studies, nine overarching factors were identified as being associated with suicide among African American adult men. The majority of factors identified were risk factors, with education and economic structure most frequently listed. Age was the only protective factor identified. The quality assessment indicated that published studies were quantitative, with the majority referencing Durkheim's theory on suicide, utilizing national data, and employing regression analyses.

Results indicate that little research exists particularly regarding suicide protective factors among African American adult men. Additionally, methodological quality enhancement may be warranted. Specific areas for future research are provided. ๑ 2008 WPMH GmbH. Published by Elsevier Ireland Ltd.

\section{Introduction}

Defined as the act of killing oneself intentionally [1], suicide is the eleventh leading cause of death among all Americans, but the sixth cause of death for African Americans aged 25-44, and the third leading cause of death for African American men aged 15-24 [2]. Although suicide rates among African Americans continue to be substantially lower than among Whites in the United States (U.S.), an increase in suicide completions has been evident in recent years, especially among men $[2,3]$. Fig. 1 depicts suicide trends from 1950-2002 specifically among African American men between the ages of 25-44, and compares these rates to those among White men in the same age category.

As also depicted in Fig. 1, among African Americans, male suicide rates (particularly for those aged 25-44) consistently exceed female suicide rates. Specifically, on average, the rate of African American men that complete suicide 
Suicide Rate Comparison: 1950-2002

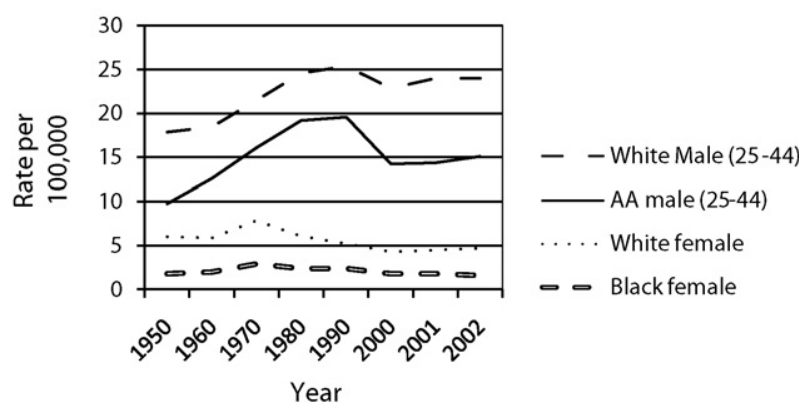

Figure 1 Suicide rate comparison: 1950-2002. In 2000, suicides were recoded from ICD-9 to ICD-10, which changed the way that they were reported. However, the trend from 2000 (when coding changed) through 2002, indicates an increasing trend of suicide among this population, especially those aged 25-44 years. Data derived from the National Center for Health Statistics (2004) [2]. AA, African American.

exceeds the rate of African American women by a factor of six, whereas nationally men complete suicide four times more often than women [4]. Perhaps because suicide rates remain lower among U.S. Blacks as compared to Whites (Fig. 1), incremental increases have gone relatively unnoticed and underresearched. Furthermore, misclassification of suicides among Blacks has been noted in the literature. Suicides are typically misclassified as accidental deaths or homicides [5], which share common risk factors with suicide and are among the top five causes of death for Black men [6,7]. This misclassification could also be the result of 'random error or nonrandom bias', impacted by cultural and economic factors (e.g. stigma, insurance benefits) which may impede the completeness of information gathered regarding the death or perhaps affect the coroner's findings [6]. Ultimately, continued misclassification may contribute to a deflation of published suicide statistics among Blacks [5] and, thus, a lack of attention and resources to this salient issue.

Although several factors have been identified as risk and protective factors for suicide [8] the majority of research, to date, has focused on Caucasian Americans. Factors that may be specific to African Americans have yet to be adequately reported in the literature. The presence of suicide as a leading cause of mortality for African American adult men, coupled with its increasing rates is cause for concern. Therefore, the purpose of this literature review is to systematically and critically analyze the available evidence regarding risk and protective factors associated with suicide among African American adult men. Suicide is a largely preventable issue [9] and a better understanding of the factors associated with suicide in this sub-population carries implications for prevention efforts, including program development and implementation.

\section{Methods}

\section{Study selection}

To fulfill the purpose of this literature review, three primary methods of selecting articles were used. The first employed a database search. Searches were performed using Cambridge Scientific Abstracts. Within Cambridge Scientific Abstracts, 14 databases including Criminology SAGE, Education SAGE, ERIC, Health Sciences SAGE, MEDLINE, NTIS, Political Science SAGE, PsycARTICLES, Psychology SAGE, PsycINFO, Social Services Abstracts, Sociological Abstracts, Sociology SAGE, and Urban Studies \& Planning SAGE were utilized to search for keywords (in the following order) with the Boolean connector, 'and': suicide, African American or Black, men or male. All studies through November 2006 were eligible for review. Based on this particular method, 266 potential journal articles were identified.

Studies were further analyzed to gauge their compliance with inclusion and exclusion criteria. Studies were included if they were: (1) Peer-reviewed, journal publications; (2) specific to Black adult men (aged 18 and older), however, articles that included women and 
other races/ethnicities were considered if results for Black men could be directly extracted; (3) empirical studies (either qualitative, quantitative, or both); (4) focused on suicide as an outcome variable; (5) performed in the United States; (6) printed in the English language. Studies were excluded if they met one or more of the following criteria: (1) Nonempirical; (2) suicide completion was not the dependent/outcome variable or the main focus, e.g., many studies emphasized suicide attempts, suicidal behavior, parasuicides, suicidality, and suicide ideology as outcomes. Articles dealing with assisted suicide (euthanasia) were also excluded; (3) age-specific results could not be extracted or the age was not within the specified range; (4) results could not be extracted for Black men; (5) studies did not occur in the United States. After applying the inclusion and exclusion criteria, the first tier yielded five articles.

The second tier of research consisted of another search, using the aforementioned databases, but modifying the keywords. The keywords, 'factors, suicide, and African American or Black' were used as the primary search terms. One new study was introduced that met the inclusion and exclusion criteria.

Thirdly, reference lists from reviewed articles were perused for potential studies. Three additional articles were identified through this process. Therefore, the final $\mathrm{N}$ was nine studies, ranging from 1974-2004 [10-18]. A matrix depicting study components was developed using the Garrard matrix method [19].

\section{Methodological quality assessment}

A systematic literature review requires a precise and methodical manner of not only summarizing the studies, but assessing the quality therein. Therefore, quality criteria were developed and assigned to further assess the articles' methodological quality (see Table 1). The inclusion of a quality assessment provides an overview of studies' rankings on key features of sound research. Previous systematic literature reviews have also employed assessments of methodological criteria $[20,21]$.

This systematic literature review particularly gauged the following aspects of the included studies: (1) the application of theory; (2) the type of research paradigm employed (qualitative, quantitative, or both); (3) the
Table 1 Quality Assessment Tool.

\begin{tabular}{ll}
$\begin{array}{l}\text { Study } \\
\text { Characteristic/Criteria }\end{array}$ & Point Specification \\
\hline $\begin{array}{ll}\text { Theoretical Framework } & \text { No: } 0 \\
& \text { Yes: } 1 \\
\text { Research Paradigm } & \text { Qualitative: 1 } \\
& \text { Quantitative: 1 } \\
& \text { Mixed-methods: } 2 \\
\text { City/county: 1 } & \text { State: 2 } \\
\text { Nata source } & \text { National: 3 } \\
& \text { Descriptives/frequencies: 1 } \\
& \text { Correlations/univariate: 2 } \\
\text { Data analysis method } & \text { Multiple/logistic } \\
& \text { regression: 3 } \\
& \text { HLM, SEM, etc: 4 } \\
& \text { Point range: 3-10 }\end{array}$ \\
\hline
\end{tabular}

$\overline{\mathrm{HLM}}$, hierarchical linear modeling; SEM, structural equation modeling.

source of data (local, state, or national); (4) the method of data analysis. These four criteria were selected because they represent components of quality research and have been used, in part, for other systematic reviews [20]. Further explanation regarding the usage of these criteria is provided below.

Theoretical frameworks can be used to explain behavior and also understand behavior change [22]. Perhaps most importantly, theory provides a record of the process utilized to conduct the research and helps facilitate replication of the process, should others desire to implement a similar one. Thus, using theory advances the field of research. Quantitative and qualitative methodologies are of tremendous utility when implemented correctly; however, both also have limitations. Thus they were awarded the same point value. A mixed methods approach was given two points, which is the sum of the qualitative and quantitative points. Data collected at a national level is generally more generalizable than data collected at the state and local levels. Therefore, research that utilized national samples was awarded the most points in the data source category (3 points), followed by state ( 2 points), and local (1 point). Finally, the points for data analysis increased with the robustness of the analysis method chosen. The total number of quality points that could be received ranged from 3-10 (see Table 1). All 
analyses (descriptive in nature) were performed using Microsoft Excel.

\section{Findings}

\section{Risk and protective factors}

Findings indicated nine overarching factors associated with suicide among adult African American men. The factors include: education, substance abuse, economic structure, marital status, age, interaction with the police, levels of violence, disease status, and geographical region. Among these nine, education and economic structure were found to be associated with suicide in African American adult men most frequently.

Two of the nine studies (22.2\%) determined an association between educational attainment and suicide. Within these studies, educational attainment was shown to be both positively and negatively related to suicide and dependent upon the age of African American men $[10,11]$. Among 25-44 year olds, those with a college education were at an increased risk of suicide. However, the relationship was reversed among those aged 55-64. In particular, those that attain a college education have lower suicide rates [11], compared to the other age categories - thus perhaps indicating a protective effect of age. Economic structure was implicated in three studies (33.3\%). Specifically, there was an inverse association between economic opportunity and suicide [12], therefore, less economic opportunity (or greater economic strain), corresponds to a heightened risk of suicide. Another study reported a positive relationship between income and Black male suicide rates [13]. A follow-up to the aforementioned study [13] found that dependence on public assistance, as a measure of economic strain, was a suicide risk factor [14]. Specifically, as dependence on public assistance increased, so did the risk for suicide completion.

Substance abuse (specifically cocaine use), geographical residence, trouble with the police, age, level of violence, marital status (specifically widowhood), and disease status were also found to be associated with suicide. Whereas a directional relationship was not sought regarding drug abuse [15], an inverse relationship was found between the percentage of Blacks dwelling in a geographical area and the nonwhite suicide rate [16]. Trouble with the police was positively associated with suicide. Simply stated, more negative contact with the police was associated with high suicide rates. Age, however, displayed an inverse association [11]. Age also seemed to mediate the effects of other variables, such as educational attainment and trouble with the police, and may be construed as a protective factor in those instances $[10,11]$. Level of violence displayed a positive association with suicide rates. Specifically, the data utilized was collected during a time of political unrest, symbolized by riots and guerilla warfare [14]. Therefore, as the number of violent events increased, so did the suicide rate among Black men. Marital status, specifically widowhood, was also found to be associated with suicide [17]. Being widowed, particularly, was associated with a nine times increased risk of suicide [17]. Disease status, specifically HIV sero-positive status, was also moderately associated with an increased risk of suicide [18].

\section{Methodological quality assessment}

Approximately half of the studies utilized theory $(55.6 \%)$. The under girding theory was fairly consistent amongst the studies, with the majority using Durkheim's theory as the basis for their research $[10,11,14]$. Others integrated Durkheim's theory with additional, more recent theory [11]. All (100\%) of the studies were quantitative. The studies analyzed data from a variety of sources at the city, county, and national levels. Specifically, three out of the nine studies (33.3\%) analyzed city or county level data $[12,15,18]$. The remaining six studies $(66.7 \%)$ analyzed national data. Data was extracted from birth and death certificates, the U.S. Census, and autopsies. The methods of statistical analysis varied, with regression being the predominate form. Approximately $33 \%$ of the studies used some form of regression, ranging from ordinary least squares regression to logistic regression $[11,13,14]$. Four $(44 \%)$ of the studies used correlational methods $[10,12,15,18]$, with the remaining two $(22 \%)$ employing descriptive methods (frequencies, etc). No studies used statistical analysis methods such as structural equation modeling. The mean number of points for this sample of studies was 6.11 (standard deviation $(\mathrm{SD})=1.7$ ), with a range 


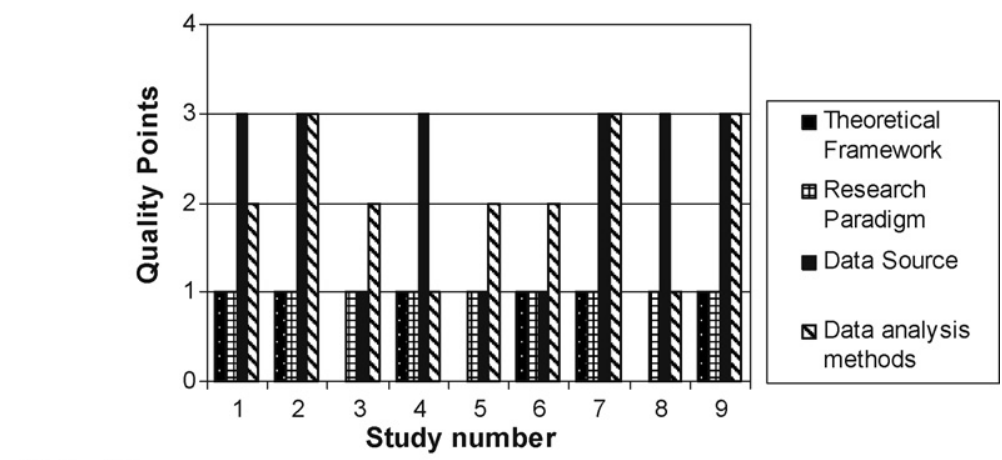

Study Kev

Study 1: Fernquist (2001) [10]

Study 5: Marzuk et al. (1997) [18]

Study 2: Fernquist (2004) [11]

Study 6: Almgren et al. (1998) [12]

Study 3: Garlow (2002) [15]

Study 7: Stewart (1980) [14]

Study 4: Luoma \& Pearson (2002) [17]

Study 8: Davis (1979) [16]

Study 9: Hamermesh (1974) [13]

Figure 2 Study Rankings on Quality Assessment Criteria.

of 4-8. The points attributed to each study (categorized by criteria) are displayed in Fig. 2 .

\section{Discussion}

\section{Risk and protective factors}

The purpose of this literature review was to systematically and critically analyze the available evidence regarding risk and protective factors associated with suicide among African American adult men. Although nine factors were identified, overall, the literature on factors associated with suicide among African American adult men is rather sparse (as evident by 9 studies in approximately 20 years). This great paucity in the literature needs to be filled - especially considering the escalating rate of suicide among African American adult men. This study revealed that the majority of available literature focuses on suicide risk factors. Within the multitude of associated risk factors, little consensus existed among the factors. A miniscule number of studies have investigated protective factors, which is interesting considering the lower rate of suicide among African Americans. This enhances the challenge of prevention efforts.

Although articles have been published regarding suicide among African Americans, few have focused specifically on African American adult men, and even fewer are empirical studies. Many available articles are theoretical or conceptual pieces [14,23], and one literature review was located [24]. While it is acknowledged that the literature review [24] provided some insight on suicide among African Americans, in general, and African American men, in particular, the current systematic review differs in five important ways: (1) it focuses strictly on factors for African American adult men and not African Americans, in general; (2) it only includes empirical studies; (3) it focuses only on suicide completion; (4) it provides a quality assessment of the limited amount of available literature; (5) it contains more recent studies (33.3\% of the studies used in this literature review were published after the Willis et al [24] study).

According to the Centers for Disease Control and Prevention, risk factors for suicide include: previous suicide attempt; history of mental disorders (particularly depression) or alcohol and substance abuse; family history of suicide or child maltreatment; feelings of hopelessness; impulsive or aggressive tendencies; barriers to accessing mental health treatment; loss (relational, social, work, or financial); physical illness; easy access to lethal methods; unwillingness to seek help because of the associated stigma; cultural and religious beliefs; local epidemics of suicide; isolation [8]. While three of these factors (physical illness, substance abuse, financial loss) may be considered consistent with the current systematic literature review, the remaining factors were not listed. 
However, many of the factors listed within the current review are congruent with the factors revealed in one of the most expansive studies of African American suicide [25]. Specifically, education, substance abuse, economic structure, marital status (although being single, not widowed), and geographical region were found to be significantly associated with suicide among African Americans compared to Whites. The similarities observed between the factors identified within this current review and the aforementioned study [25] can perhaps be attributed to the overwhelmingly male (approximately 87\%) composition of that study's [25] sample. Although the sample was overwhelmingly male [25], that study was excluded from this review because the results were for African Americans, in general, without clear delineation of the factors solely relating to African American males.

Economic structure was also identified as a factor associated with suicide, however, other factors besides economic strain must also influence the relationship [26]. This is due to the following rationale - if the relationship was direct and consistently inverse, African Americans as a group (with higher unemployment rates, lower median incomes, etc) should have higher rates of suicide compared to Whites [26]. However, data indicates that African American suicide rates are consistently lower (Fig. 1). In the absence of a consensus, more research is needed to understand this anomaly. Several other studies identified contributory factors congruent with those in the current review (e.g. income equality, disadvantage), but the age range included adolescents, thus causing them to be eliminated from this review $[27,28]$.

Although we were able to gain information about risk factors, less is known about protective factors for African American adult men. Currently, general population protective factors include effective clinical care; easy access to clinical interventions; social support; skills (problem-solving, conflict resolution, nonviolent handling of disputes); cultural and religious beliefs [8]. There are studies that highlight protective factors among African American women who attempt suicide [29], but this systematic literature search reveals that many of these factors have not been examined for African American adult men that have completed suicide.

\section{Areas for future research}

Based on the findings from this study, the following are suggested as areas for future research:

- More research investigating risk and protective factors. A miniscule number of studies regarding suicide among African Americans exists; and researchers lament that suicide is 'poorly understood' in this sub-population [30]. The current review echoes these sentiments by demonstrating that there is a scarcity of research concerning suicide risk and protective factors among African American adult males. In addition to the litany of general population suicide risk and protective factors [8], emerging research suggests that being an immigrant, versus a native-born Black person in America, may exert a protective effect [31]. Further investigation is required to prevent researchers and practitioners from prematurely and, perhaps, erroneously applying the aforementioned factors to this sub-population.

- Additional studies empirically investigating socio-cultural forces and the effect of subsequent interventions. The influence of socio-cultural influences, such as racism, has been under-investigated, empirically, in suicide completions. Racism is identified as the 'missing variable' that can impact psychological functioning, as well as socioeconomic status, etc, that may result in the differences witnessed in certain health indicators [32]. Although studies are emerging concerning racism and suicide attempts [33], additional research is needed regarding suicide completions. Additionally, studies have noted that the majority of known risk and protective factors have been based upon Whites [25], resulting in interventions predicated upon these factors and this sub-population. However, findings from the current systematic literature review suggest that differences exist between the known general population risk and protective factors, and the factors that are relevant for African American males. Therefore, this study reinforces the need for additional research regarding factors associated with suicide, as well as suicide prevention interventions, for this particular sub-population. Moreover, there is a growing body of literature that calls for 
culturally appropriate interventions to effectively address suicide among African Americans. For example, in one study, African Americans who committed suicide were more likely to frequent church services compared to Whites [25]. This suggests a potential partner for research and intervention. However, more research must be conducted to assess the feasibility and impact of such socio-cultural interventions for African Americans, in general, and, more specifically, African American males.

- More qualitative research. All (100\%) of the studies were quantitative. While quantitative research has its merits, researchers may want to also explore qualitative methods, such as case studies. This method was used with the wife of a deceased White male who committed suicide [34]. The wife was able to provide invaluable information concerning her husband's behavior prior to his suicide. Methodologically, however, it may be more difficult to ascertain a participant pool for qualitative studies in the African American community - which has historically neglected and stigmatized mental health [35].

- Utilization of diverse theory. In 1978 , the following was stated: 'it has been evident for several decades that we need a truly psychosocial approach, not an amalgam of Freud and Durkheim, but one that examines the psychodynamics of suicide of differing groups' [36]. However, the majority of studies after publication of this statement have primarily employed Durkheim's theory. Durkheim undoubtedly laid the foundation in this particular research arena, but additional theory may provide further insight into suicide among African American adult men.

- Employment of more robust analyses. Multilevel modeling, such as structural equa- tion modeling and hierarchical linear modeling, could contribute to an explanatory or predictive framework regarding suicide among African American men. Hierarchical linear modeling will allow testing at various levels, including the individual, county, and national level, for example. These methods will allow researchers to build upon prior work and test some of the factors that have been shown to be empirically associated with suicide among African American adult men.

\section{Limitations}

Despite efforts to ensure thoroughness of this literature review, it possesses several limitations. The review only included those factors associated with completed suicide, which inherently requires that the sample be deceased. However, it is acknowledged that suicide attempts, suicide ideation, etc could be critical to the prevention of suicides. These were beyond the scope of this investigation. It is further acknowledged that there is a body of literature that deals with overall mental health and other mental health issues, such as depression, as outcomes. However, this particular literature review sought to delineate those factors that are directly associated with suicide as an outcome. Although the authors sought to include all articles that met the search criteria, issues such as database variation and indexing of journals may have contributed to the omission of relevant articles from this review. Undoubtedly, additional and/or different criteria could have been included to assess the methodological quality of the studies. Additionally, inter-rater reliability was not established which may have strengthened the coding of factors.
References ${ }^{1}$

[1] Guralnik DB, editor. Webster's New World Dictionary of the American Language. 2nd edition New York: Simon \& Schuster; 1986.

[2] National Center for Health Statistics. Health, United States, 2004, with Chartbook on Trends in the Health of Americans. Hyatts- ville, MD: Centers for Disease Control and Prevention, National Center for Health Statistics; 2004. Available at: http://www. cdc.gov/nchs/data/hus/hus04.pdf.

[3] Centers for Disease Control and Prevention: National Center for Injury Prevention and Control. Suicide: Facts at a Glance. Atlanta,
GA: CDC; 2008. Available at: http:// www.cdc.gov/ncipc/dvp/suicide/suicide_data_sheet.pdf.

[4] Kochanek KD, Murphy SL, Anderson RN, Scott, C. Deaths: Final Data for 2002. National Vital Statistics Reports, vol. 53, no. 5. Hyattsville, MD: National Center for Health

\footnotetext{
1 * denotes studies that were analyzed for this review.
} 
Statistics; 2004. Available at: http://www.cdc. gov/nchs/data/nvsr/nvsr53/nvsr53_05.pdf.

[5] Anglin DM, Gabriel KO, Kaslow NJ. Suicide acceptability and religious well-being: a comparative analysis in African American suicide attempters and non-attempters. J Psychol Theol 2005;33(2):140-50.

[6] Mohler B, Earls F. Trends in adolescent suicide: misclassification bias? Am J Public Health 2001;91:150-3.

[7] Centers for Disease Control and Prevention. Men's Health. Leading Causes of Death in Males: United States, 2004. All Males by Race/Ethnicity - United States, 2004. Atlanta, GA: CDC. Available at: http:// www.cdc.gov/men/lcod/index.htm.

[8] Centers for Disease Control and Prevention: National Center for Injury Prevention and Control. Understanding Suicide: Fact Sheet. Atlanta, GA: CDC; 2008. Available at: http:// www.cdc.gov/ncipc/pub-res/suicide_factsheet2008.pdf.

[9] United States Department of Health and Human Services. National Strategy for Suicide Prevention: Goals and Objectives for Action. Rockville, MD: U.S. Department of Health and Human Services, Public Health Service; 2001. Available at: http://download.ncadi.samhsa.gov/ken/pdf/SMA013517/SMA01-3517.pdf.

[10] *Fernquist RM. Education, race/ethnicity, age, sex, and suicide: individual-level data in the United States, 1991-1994. Curr Res Soc Psychol 2001;6(18):277-90.

[11] *Fernquist RM. Educational attainment and the payoff of education: black male suicide in the United States, 1947-1998. Curr Res Soc Psychol 2004;9(13):184-93.

[12] *Almgren G, Guest A, Immerwahr G, Spittel M. Joblessness, family disruption, and violent death in Chicago, 1970-90. Soc Forces 1998;76(4):1465-93.

[13] *Hamermesh DS. The economics of Black suicide. South Econ J 1974;(41):188-99.

[14] * Stewart JB. The political economy of Black male suicides. J Black Stud 1980;11(2): 249-61.
[15] * Garlow SJ. Age, gender, and ethnicity differences in patterns of cocaine and ethanol use preceding suicide. Am J Psychiatry 2002;159(4):615-9.

[16] *Davis R. Black suicide in the seventies: current trends. Suicide Life Threat Behav 1979;9(3):131-40.

[17] *Luoma JB, Pearson JL. Suicide and marital status in the United States, 1991-1996: is widowhood a risk factor? Am J Public Health 2002;92(9):1518-22.

[18] *Marzuk PM, Tardiff K, Leon A, Hirsch CS, Hartwell N, Portera L, et al. HIV seroprevalence among suicide victims in New York City. Am J Psychiatry 1997;154(12):1720-5.

[19] Garrard J. Health Sciences Literature Review Made Easy: The Matrix Method. Gaithersburg: Aspen; 1999.

[20] Meert AP, Paesmans M, Martin B, Delmotte $P$, Berghmans $T$, Verdebout JM, et al. The role of microvessel density on the survival of patients with lung cancer: a systematic review of the literature with meta-analysis. Br J Cancer 2002;87(7):694-701.

[21] Goodson P, Buhi E, Dunsmore SC. Selfesteem and adolescent sexual behaviors, attitudes, and intentions: a systematic review. J Adolesc Health 2006;38(3):310-9.

[22] Glanz K, Lewis FM, Rimer BK, editors. Health Behavior and Health Education: Theory, Research, and Practice. 2nd edition. San Francisco: Jossey Bass; 1997.

[23] Washington RO, McCarley LD. A postmodern perspective on Black suicides in the United States. J Hum Behav Soc Environ 1998;1(2):225-42.

[24] Joe S, Kaplan MS. Suicide among African American men. Suicide Life Threat Behav 2001;(31):106-21.

[25] Willis LA, Coombs DW, Drentea P, Cockerham WC. Uncovering the mystery: factors of African American suicide. Suicide Life Threat Behav 2003;33(4):412-29.

[26] Stack S. The relationship between culture and suicide: an analysis of African Americans. Transcult Psychiatry 1998;35(2): 253-69.
[27] Burr JA, Hartman JT, Matteson DW. Black suicide in US metropolitan areas: an examination of the racial inequality and social integration-regulation hypotheses. Soc Forces 1999;77(3):1049-80.

[28] Kubrin CE, Wadsworth T, DiPietro S. Deindustrialization, disadvantage, and suicide among young Black males. Soc Forces 2006;84(3):1559-79.

[29] Nisbet PA. Protective factors for suicidal Black females. Suicide Life Threat Behav 1996;26(4):325-41.

[30] Castle K, Duberstein PR, Meldrum S, Conner $K R$, Yeates C. Risk factors for suicide in Blacks and Whites: an analysis of data from the 1993 National Mortality Followback Survey. Am J Psychiatry 2004;161(3):452-8.

[31] Singh GK, Miller BA. Health, life expectancy, and mortality patterns among immigrant populations in the United States. Can J Public Health 2004;95(3):14-21.

[32] Smith GD, Neaton JD, Wentworth D, Stamler R, Stamler J. Mortality differences between black and white men in the USA: contribution of income and other risk factors among men screened for the MRFIT. Lancet 1998;351(9107):934-9.

[33] Rowell K, Rivers D. Racism as a stressor among African American suicide attempters: examining gender disparities. Eta Sigma Gamma Student Monogr 2006; 23(2):30-6.

[34] Kewman DG, Tate DG. Suicide in SCl: a psychological autopsy. Rehabil Psychol 1998;43(2):143-51.

[35] United States Department of Health and Human Services, Mental Health. Culture, Race, and Ethnicity - A Supplement to Mental Health: A Report of the Surgeon General. Rockville, MD: U.S. Department of Health and Human Services, Public Health Service, Office of the Surgeon General; 2001. Available at: http://www.surgeongeneral.gov/ library/mentalhealth/cre/.

[36] Hendin H. Suicide: the psychosocial dimension. Suicide Life Threat Behav 1978;8(2): 99-117. 